\title{
ESTRATEGIAS DE MERCADOTECNIA SOCIAL $Y$ SU CONTRIBUCIÓN EN LA FIDELIZACIÓN DE CLIENTES DE LAS AGENCIAS DE LA ONG PRO MUJER DEL CERCADO DE AREQUIPA, AÑO 2014
}

\section{Social marketing strategies and their contribution to customer loyalty agencies NGO pro fencing woman Arequipa, 2014}

Alcides Gutiérrez Gómez

\section{RESUMEN}

Objetivo: La presente investigación tuvo como objetivo determinar si las estrategias de mercadotecnia social, han contribuido en la fidelización de clientes de las agencias de la ONG pro mujer del cercado de Arequipa, año 2014.

Método: El tipo de investigación es aplicada. Es de naturaleza descriptiva, y se midió las percepciones de los clientes de la ONG Pro Mujer del cercado de Arequipa, respecto a las estrategias de mercadotecnia social utilizadas en el año 2014. También es correlacional, por lo que se analizó la relación entre las estrategias de mercadotecnia social y la fidelización de los clientes de la ONG Pro Mujer del cercado de Arequipa en el año 2014. Para esto, se encuestaron a 140 clientes de las agencias de la ONG Pro Mujer del cercado de Arequipa, del año 2014, utilizándose un cuestionario de 22 preguntas dicotómicas (estrategia mercadotecnia social) y 22 de escala de Lickert (fidelización de clientes), evaluando en cada bloque de preguntas los respectivos indicadores planteados en la investigación.

Resultados: Para comprobar la contribución de cada estrategia, se procedió con la prueba estadística no paramétrica del chi cuadrado, que determinó, que la estrategia "producto" si contribuye en la fidelización de clientes, al obtener un valor-P de 0,0498; y las estrategias "precio", "promoción" y "distribución" no contribuyen en la

\footnotetext{
${ }^{5}$ Magister en Administración y Dirección de Empresas
}

fidelización de clientes al obtener el Valor-P de $\quad 0,2660, \quad 0,7942$ y 0,7286 respectivamente.

Conclusión: Las estrategias de mercadotecnia social de las agencias de la ONG Pro Mujer del cercado de Arequipa, en el año 2014, no han contribuido en la fidelización de sus clientes.

Palabras claves: mercadotecnia social, grupo solidario, fidelización de clientes.

\section{ABSTRACT}

Objective: This study aimed to determine whether social marketing strategies have contributed to the loyalty of the NGO agencies pro fencing woman Arequipa, 2014.

Method: The research is applied. It is descriptive in nature, so that customer perceptions of the NGO Pro Mujer fencing of Arequipa, on strategies of social marketing used in 2014. It is also correlational, was measured so that the relationship between analyzed social marketing strategies and customer loyalty NGO Pro Mujer fencing of Arequipa in 2014. for this, customers surveyed 140 agencies NGO Pro Mujer fencing of Arequipa, 2014, using a questionnaire of 22 dichotomous questions (social marketing strategy) and 22 Likert scale (customer loyalty), evaluating each block of the indicators raised questions in the investigation.

Results: To test the contribution of each strategy, proceeded to perform the nonparametric statistical test of chi square, which determined that the strategy "product" if it contributes to customer loyalty, to obtain a value-P 0.0498 ; and 
strategies "price", "promotion" and "distribution" does not contribute to customer loyalty to obtain the p-value of $0.2660,0.7942$ and 0.7286 respectively.

Conclusion: Strategies social marketing agencies NGO Pro Mujer fencing of
Arequipa, in 2014, have not contributed to the loyalty of its customers.

Keywords: social marketing, solidarity group, customer loyalty.

\section{INTRODUCCIÓN}

Desde décadas pasadas operan en el Perú los Organismos No Gubernamentales y en la actualidad se encuentran bajo la supervisión de la (Agencia Peruana de Cooperación Internacional, 2007),y esta las define como instituciones privadas sin fines de lucro, con una estructura y política de dirección autónoma, independiente del Gobierno y de las instituciones estatales; este es el caso de la ONG Pro Mujer que fue fundada en el año 1990 en Bolivia, y en el Perú inició operaciones en el año 1999 en la ciudad de Puno, en base a los conocimientos y la experiencia adquirida en Bolivia y Nicaragua, desde entonces la ONG Pro Mujer en el Perú ha seguido creciendo, sirviendo y empoderando a las mujeres pobres en el país, para ello cuenta con 41 agencias distribuidas en las regiones de Puno, Arequipa, Moquegua, Tacna, Cusco, Abancay, Cajamarca, Ancash y Huánuco.

Siendo la preocupación de los responsables de las agencias de la ONG Pro Mujer del cercado de Arequipa: Avelino, Altiplano, Zamacola y Paucarpata ( $70 \%$ de créditos en la región), la fidelización de sus clientes, a través de una relación estable y duradera, siendo la percepción de la población sobre la ONG Pro Mujer de considerarla como una Institución micro financiera, a pesar de no estar bajo la supervisión de la Superintendencia de Banca, Seguros y AFP, y así también reporta a la central de riesgo Infocorp el historial crediticio de sus clientes, por lo que las otras entidades del sector financiero y micro financiero acceden a estos datos e identifican a clientes de muy buena calificación crediticia y son captados a través de la oferta de productos financieros atractivos, principalmente la tasa de interés, viéndose tentados los clientes de la ONG Pro Mujer de cambiarse a otra entidad crediticia.

En un mercado caracterizado por la competencia, las organizaciones utilizan una serie de herramientas en la gestión, en este caso hacemos referencia a las estrategias de mercadotecnia social que ejecuta la ONG Pro Mujer en las agencias del cercado de Arequipa, a través de la estrategia del "producto" como son:los préstamos a través de los grupos solidarios conformados por 4 a 8 socias, las cuales constituyen una garantía grupal al momento de otorgarles un préstamo, asimismo se les brinda capacitación empresarial y de empoderamiento, educación de salud preventiva, servicios primarios de salud de alta calidad a bajos costos; la estrategia del "precio" que se refiere al costo financiero por los préstamos que realiza y por los servicios de salud y capacitación empresarial; la estrategia de "promoción" de los diferentes servicios que presta y los medios de difusión que utiliza y finalmente la estrategia de "distribución" refiriéndose a las ventajas o desventajas que trae al cliente la ubicación de las agencias y puntos de atención de la ONG Pro Mujer en el cercado de Arequipa.

Actualmente cada sociedad debe enfrentar y resolver diversas problemáticas sociales. Para ellos existen diversas alternativas a través de los cuales se tratan de erradicar males como el alcoholismo, drogadicción y enfermedades crónicas degenerativas, entre otras. Uno de los caminos es la mercadotecnia social, que ha sido diseñada para implementar programas que promueven cambios de conducta que beneficien la sociedad (Grier \& Bryant, 2004).

Para (Kotler \& Roberto, Marketing social: estrategias para cambiar la conducta pública, 1992), las campañas de cambio social se han enfocado en diversos campos que son de importancia para la sociedad, los campos en los que se enfocan son: 
- Reformas sanitarias (tabaquismo, abuso de drogas, nutrición, mejoramiento de la condición física, etc.).

- $\quad$ Reformas del medio ambiente (cuidado del agua, aire limpio, conservación de los parques y bosques, protección de la flora y la fauna, etc.).

- $\quad$ Reformas educativas (aumento en la alfabetización de los adultos, mejorar las escuelas públicas, etc.).

- Reformas económicas (revitalización de ciudades antiguas, atracción de inversión extranjera, etc.).

El diseño del producto social, se realiza luego de la investigación y el análisis de la sociedad. Ese es el punto clave donde se apoyarán la futuras acciones y actividades de Marketing, ante todo deben detectarse las necesidades de los destinatarios, así poder satisfacerlas. Antes de lanzar el producto social, es necesario hacer una prueba previa, así ver que factibilidad tiene que sea adoptado. Es muy importante en esta etapa, indagar si el público objetivo entiende el concepto del producto social ofrecido, si ve los beneficios, si a estos beneficios los considera importantes y si adoptaría por el producto social que se le ofrece. Por otro lado, se busca estimular a ese grupo a que aporte sugerencias, y cuente que tipos de sacrificios está dispuesto a hacer por adoptar el producto social.

Cada producto social atiende un determinado tipo de Demanda, al respecto los distintos tipos de demanda son los siguientes:

a) Demanda latente: Descubrir una necesidad o demanda latente, presenta la oportunidad de introducir un nuevo producto social. La demanda es latente, cuando un considerable número de personas comparte una fuerte necesidad de un producto o servicio social efectivo que no existe, como en el antitabaquismo o el control de la contaminación. La tarea de los expertos, es transformar la demanda latente en demanda real por medio de un producto o servicio eficaz.

b) Demanda insatisfecha: En este caso, los productos o servicios disponibles no son suficientes ni satisfacen la demanda de los destinatarios. Aquí, los especialistas deberán lograr el desarrollo de un nuevo producto o mejorar uno ya existente para colmar la brecha; cuando traten de satisfacer una demanda insatisfecha tendrán que distinguir entre una brecha en el nivel de satisfacción y un vacío en el tipo de satisfacción. La brecha en la clase de satisfacciones, se presenta cuando los destinatarios pueden obtener satisfacción, solo por medio de un producto mejorado o un nuevo producto que corrija las deficiencias de los existentes; el vacío en el nivel de satisfacción tiene que ver con una situación donde los productos de que se dispone no pueden brindar el nivel, grado o satisfacción deseados

c) Demanda dañina: Es cuando los destinatarios tienen ideas socialmente perjudiciales, como por ejemplo el racismo, o tienen hábitos como tomar demasiado alcohol o conducir imprudentemente. Para lograr que estos grupos abandonen esas prácticas dañinas, los especialistas deben proporcionar una idea o práctica sustitutiva satisfactoria; un error estratégico muy común, es el de intentar de introducir un sustituto totalmente opuesto a la idea o conducta indeseable.

d) Demanda dual: Es cuando tienen que crearse destinatarios, tanto para un concepto de producto (antitabaquismo) como un instrumento o medio para realizar el valor de producto social (producto tangible: pastilla para quitar la ansiedad de fumar).

e) Demanda abstracta: Se presenta, cuando en una campaña de marketing social tratan de lograr solamente, la adopción de una idea. Si bien todos los programas buscan que 
los destinatarios adopten una idea, de lo que se trata aquí, es de limitar el objetivo durante un tiempo, para difundir la conciencia pública sobre un problema social, como por ejemplo fue el caso del Proyecto Hambre, una organización sin fines de lucro fundada en 1977. El objetivo del Proyecto Hambre fue eliminar el hambre en el mundo, pero la organización no dispone los recursos suficientes para hacer que cada nación emprenda acciones para acabar con el hambre. Entonces la campaña se ocupó de actuar para generar el compromiso de eliminar el hambre. Para alcanzar esa meta, se desarrollan programas en las que se difunden fotos educativas, material impreso y electrónico, acontecimientos transmitidos para llamar la atención de los líderes de opinión, educadores, diseñadores de políticas y especialistas en desarrollo.

f) Demanda irregular: La donación de sangre necesita de donantes que generalmente colaboran de manera ocasional, es decir irregular. En este caso, se deben encontrar nuevas formas de atraer nuevos donantes de sangre.

g) Demanda vacilante: Este tipo de demanda se puede presentar, si luego de lanzado un producto con éxito, la demanda del producto social comienza a debilitarse. En ese caso, se debe poner más atención en volver a lanzar el producto con un nuevo programa de marketing social que apoye el nivel deseado de demanda. Luego de determinar el tipo de demanda, se debe realizar la segmentación al que se quiere dirigir el programa que utilice el marketing social.

De acuerdo con (Weinrich, 1999), la mercadotecnia social es el uso de técnicas de mercadotecnia comercial para promover la adopción de un comportamiento que esté a favor del bienestar de la audiencia objetivo o de la sociedad, en ambas disciplinas se pueden apreciar la implementación de los métodos que utilizan las grandes compañías, las cuales están enfocados en sus consumidores, investigaciones de mercados así como en un proceso sistemático para el desarrollo de programas de mercadotecnia, lo que diferencia a ambas disciplinas está en que, en la mercadotecnia social los beneficios se acumulan para el individuo o la sociedad, fuera de que el beneficiario sea la organización anunciante. De ahí que los sectores que hacen uso de la mercadotecnia social sean:

- $\quad$ ONG'S (Organizaciones No Gubernamentales).

- Dependencias de Gobierno.

- Instituciones no lucrativas.

- Universidades.

- $\quad$ Fundaciones.

- Asociaciones civiles.

- Agencias de servicio social, etc.

La mercadotecnia comercial ha sido definida por varios autores de entre los cuales se puede mencionar la propuesta de (Kotler, Dirección de Mercadotecnia, 2001), la cual dice que la mercadotecnia es un proceso social y administrativo mediante el cual grupos e individuos obtienen lo que desean a través de generar, ofrecer e intercambiar productos de valor con sus semejantes.

(Stanton, Etzel, \& Bruce, 2004), proponen la siguiente definición de marketing:"El marketing es un sistema total de actividades de negocios ideado para planear productos satisfactores de necesidades, asignarles precios, promover y distribuirlos a los mercados meta, a fin de lograr los objetivos de la organización. 
Tomando en cuenta lo anterior, la mercadotecnia comercial es una disciplina que se encarga de la satisfacción de las necesidades de los individuos por medio del desarrollo de una estrategia que nos permite promover un intercambio entre las organizaciones que producen productos y servicios con aquellos que los necesitan (clientes) para que se logren satisfacer las necesidades de cada individuo.

Dentro de la mercadotecnia social y comercial se puede diferenciar la existencia de un vínculo muy estrecho entre ambas disciplinas ya que el origen de la primera proviene del segundo concepto, pero debido a sus áreas de aplicación presentan diferencias muy notorias al momento de su implementación ya que obedecen a fines distintos (Kotler \& Zaltman, Marketing Social: Una aproximación al cambio social planificado, 1971).

La aparición del marketing y de la tendencia hacia la satisfacción máxima de los consumidores ha generado algunos excesos que en el mediano plazo pueden ser nocivos para la sociedad. Así por ejemplo, con el fin de facilitar el consumo, las empresas han incrementado la utilización de embalajes desechables o la utilización de productos nocivos que pueden ser un grave problema para el medio ambiente. Esta situación ha generado una reacción social importante, que ha llevado a numerosas empresas a plantear limitaciones a su política de satisfacción de sus consumidores. Por lo tanto, muchas de ellas actualmente hacen hincapié en su orientación hacia la protección de los intereses de la sociedad antes que la satisfacción de los intereses individuales de sus consumidores. El concepto subyacente de esta posición es conocido como orientación al marketing social. "Los consumidores van a preferir aquellas empresas que los protegen en el largo plazo (como miembros de la sociedad), en lugar de aquellas empresas que se preocupan únicamente por su bienestar inmediato" (Arellano, 2010).

Para desarrollar el marketing social de las ONG's son muy útiles las siguientes recomendaciones del prestigioso autor estadounidense Alan Andreasen, citado por (Mendive, Marketing Social, 2011)

a) Se debe investigar y analizar al público a quien se va a dirigir la estrategia de marketing social para conocer sus necesidades, deseos, percepciones actitudes y deseos. Por ejemplo: los jóvenes, adultos, ancianos, etc.

b) Tener siempre presente al público objetivo al que se quiere influir en sus pensamientos y sus acciones, en todas las etapas del programa.

c) Diseñar una apropiada presentación del comportamiento a promover para que tenga significado y pueda causar un buen impacto en el público objetivo.

d) Realizar una continua revisión del programa para poder mejorarlo

Cada ONG debería considerar que cuando promueve un cambio de comportamiento, debe competir con otros patrones de comportamiento no deseable y que también compite con otras ONG'S o inclusive organismos de gobierno, como por ejemplo los programas de lucha contra la drogodependencia, donde existes programas diseñados por distintas ONG's y programas implementados por organismos estatales nacionales, provinciales y municipales. Por eso es muy importante, que en una primera etapa se dedique tiempo en informar y educar para que se comprenda mejor el mensaje y se entienda porque es mejor vivir adoptando el cambio de comportamiento propuesto.

Posteriormente, se debe inducir a la acción ayudando al público-objetivo a responder a los siguientes interrogantes:

a) ¿Cuáles serán los beneficios de comprometerme con este nuevo comportamiento?

b) ¿Qué costo me demandara este cambio?

c) ¿Qué personas importantes para mí, están necesitando que lo haga? 
d) ¿Estoy en condiciones de llevarlo a cabo?

Ante estas cuestiones, los programas de marketing social deben hacer hincapié en que el cambio es socialmente deseable y fácil de hacer. Andreasen, citado por (Mendive, Marketing Social, 2011), menciona las principales estrategias que el profesional de marketing social o sociotecnia, puede utilizar para hacer que la población se comprometa con el cambio que se promueve:

a) Destacar muy especialmente los beneficios.

b) Minimizar los costos del cambio.

c) Señalar la necesidad de urgente cambio, para beneficio de la sociedad

d) Enseñar a los destinatarios para que estén en mejores condiciones de actuar

e) Trabajar para que disminuya el deseo por las alternativas que compiten con el cambio deseado.

La elección de una u otra alternativa depende de lo siguiente: de observar donde está el público-objetivo situada en ese momento y de la evaluación de las ventajas que existan para cada alternativa. Por eso son tan importantes las tareas de investigación, que deben acompañar al programa de marketing social en todas sus etapas.

La empresa está y ha estado siempre orientada al cliente. Su objetivo consiste en satisfacer las necesidades que los individuos tienen a lo largo de su vida, para así lograr que ésta sea cada vez más plena y placentera. En ese contexto, con mayor frecuencia, las empresas se preocupan por extender sus objetivos y estrategias comerciales hasta el punto de que el cliente deposite confianza en ellas de forma casi inconsciente. Para alcanzar tales metas no es suficiente una calidad en el producto o servicio que se vende, sino que es imprescindible el bienestar psicológico en la acción de vender. Aquí en la coherencia de la satisfacción total material y espiritual del cliente, encuentra el empresario la razón de la atención al cliente (Bastos, 2006).

Al respecto (Fidelizarclientes.es, 2014), señala que actualmente conseguir nuevos clientes cada vez es más costoso y difícil como consecuencia de consumidores más informados, exigentes y sofisticados, el acortamiento del ciclo de vida de los productos, la hiper segmentación de los mercados, mercados más competitivos, competidores más globales, exceso de oferta sobre demanda, la fragmentación de los medios de comunicación, la mayor fuerza de negociación de los distribuidores, el desarrollo de las marcas propias de la distribución y la disminución de la notoriedad de las marcas.

Las causas que provocan la pérdida de los clientes son:

$\begin{array}{lll}\text { - } & \text { Defunción } & 1 \% \\ \text { - } & \text { Traslado de domicilio } & 3 \% \\ \text { - } & \text { Influencia de amistades } & 5 \% \\ \text { - } & \text { Acciones competidores } & 9 \% \\ \text { - } & \text { Insatisfacción con el producto } & 14 \% \\ \text { - } \quad \text { Insatisfacción con el servicio } & 68 \%\end{array}$

Si una empresa pierde un $20 \%$ de sus clientes y no consigue captar nuevos, en cinco años habrá desaparecido. Por ello, la importancia que tiene en nuestros días la retención de los 
clientes. Pero, ¿qué es la fidelidad?. Un cliente fiel es aquel que repite las compras de una determinada marca. La fidelidad implica el mantenimiento de relaciones sólidas a largo plazo.

(Barquero, 2007), refiere a que la implantación de medidas de fidelización generalmente lleva consigo una fuerte carga de incertidumbre y el director de marketing suele enfrentarse a un numeroso grupo de escépticos acostumbrados a las acciones de toda la vida.

Además, cualquier acción de fidelización supone un importante desembolso cuyos resultados son fácilmente medibles y contrastables con los recursos empleados. Otro tipo de acciones (de atención, de oferta de valor) suelen pasar más inadvertidas. Pero un programa de fidelización será observado con lupa por toda la compañía y, en muchos casos, desatará euforias iniciales que deberemos contener para no crear falsas expectativas.

Por ello, los objetivos de la fidelización deben dejarse bien claros a todas las personas involucradas. Es necesario dar a entender que lo que se busca no es únicamente aumentar el volumen de las ventas o prestación de servicios.

Sino además, se pretende generar que las empresas se solidifiquen en un mediano plazo. Para esto hay que tomar ciertos aspectos que aportará un buen modelo de fidelización:

- Fidelizar a los mejores clientes con un reconocimiento de su importancia (se debe de tomar en cuenta que un plan de fidelización no es para todos)

- Mejorar la oferta de valor, introduciendo nuevos servicios involucrados al programa de fidelización.

- Obtener mayor y mejor información de los clientes, ya que si se usa una tarjeta de fidelización, ésta debe de ser inteligente y se pueda recopilar toda la información posible del cliente.

- Establecer un nuevo canal de relación y comunicación con los clientes.

- Detectar posibles acciones de la competencia sobre la cartera de la empresa.

De acuerdo al autor (Barquero, 2007), "existen dos modos básicos degenerar fidelización. El primero de ellos, es más simple, más cercano al marketing de producto y a todas luces menos efectivo por la facilidad de réplica, es el de programas de fidelización; mientras que el segundo es el de la fidelización intrínseca al servicio. Es más complicado de aplicar, pero a la larga es el que mejor efectividad proporciona. La fidelización puede implementarse de forma directa, mediante programas que informan a los clientes, o bien de forma implícita, mediante la propia prestación del servicio".

La fidelización del cliente es una tarea de vital importancia para la supervivencia de la empresa. La mayor parte de las carteras de clientes se crean en función de las previsiones que se deducen de estos hábitos en los clientes. Permite a las empresas especializar sus productos, ya que saben exactamente a quién dirigirse. A través de encuestas y otros estudios de posventa, se obtiene información válida para la realización de mejoras en los atributos de estos productos. El cliente fidelizado proporciona estabilidad a la empresa, que puede organizar mejor su contabilidad e inversión, arriesgando en menor medida, ya que es más fácil establecer objetivos realistas. La fidelización sirve a las organizaciones para elevar el nivel de servicio en relación con sus competidores, ya que son conscientes de la cuota de mercado que ocupan y la que desea alcanzar (Bastos, 2006, pág. 14).

Al respecto (Martínez-Vilanova, 2004), se refiere a la satisfacción como el paso previo a la repetición e, incluso, a la prescripción del producto o servicio a otros posibles compradores. Sin embargo, se sabe que muchos clientes, que se consideran satisfechos, cambian de 
proveedor. Se puede afirmar que la lealtad es la única manera de conseguir beneficios sostenibles para una empresa, de aquí que el marketing relacional, centrado en la satisfacción del cliente como individuo, tenga cada vez mayor importancia para las empresas.

La ansiada fidelización de la clientela requiere de un esfuerzo por parte de toda empresa y esta dificultad es más o menos alta dependiendo de:

- El número de competidores que ofrecen el mismo producto o servicio y la homogeneidad existente entre ellos. Como es lógico, a mayor oferta y menos diferenciada la probabilidad de perder un cliente que cambie de suministrador aumenta, ya que pueden elegir de entre muchos productos o servicios similares. Estas pérdidas serán aún mayores si el producto o servicio tienen un precio bajo y si se derivan consecuencias poco importantes en caso de una calidad escasa. El producto o servicio está amparado por una fuerte imagen de marca y, sobre todo, cuando el público es muy sensible hacia esa imagen. Este es el caso de productos muy prestigiados; la fidelidad aumenta cuando las consecuencias de elegir mal al proveedor son importantes, como es el caso de una clínica prestigiosa o un despacho de abogados de fama.

- Los costes de cambio son altos, por lo que un usuario de un sistema de calefacción que consume gas propano, difícilmente comprará una nueva caldera que utilice el gasóleo, ya que deberá construir un nuevo depósito para éste y su inversión en obra es demasiado grande, su opción más lógica será continuar con su sistema de gas.

- La atención y, en general el trato proveedor-cliente, constituyen una parte importante del producto o servicio adquirido, este es el caso de los pequeños hoteles que tienen clientes habituales, los cuales difícilmente cambiarán de alojamiento si se ha conseguido una relación personal que haga que aquellos se sientan como en casa.

- La relación precio/valor, si el consumidor dispone de otra oferta que, a su juicio, le proporciona más por menos, la posibilidad de que cambie es alta.

(Barquero, 2007), define tres tipos de programas de fidelización:

$>\quad$ De Recompensa, son los más usuales, son muy similares al descuento por volumen que se aplica desde tiempo inmemorial, aunque algo más elegantes. Ejemplos básicos son los programas de puntos canjeables por regalos, envío de bonos por descuento al superar cierto volumen de consumo, entrega de premios relacionados con el nivel de compras, etc. Estos programas si realmente se quieren centrar en el cliente deben de tomar en cuenta los hábitos, gustos y preferencias de éste.

$>\quad$ De servicios exclusivos, estos programas son más sofisticados, por ello se enfocan más en los clientes. Es clave que el programa de fidelización sea transparente y que todos los usuarios tengan conocimiento sobre el porqué un cliente logra el estatus de VIP así como lo que hay que hacer para alcanzar dicho estatus. Ser cliente VIP accede a determinados servicios que lo hacen exclusivo.

De invitación a eventos, otro valioso programa de fidelización es el de invitación a eventos, el cual consiste en invitar a los mejores clientes a determinados eventos, ya sean conciertos, fiestas, ferias o algún otro. Este programa es un elemento extraordinario de reconocimiento del valor que tiene el cliente para la empresa.

\section{MATERIALES Y MÉTODOS}

El tipo de investigación es aplicada. El diseño de investigación es descriptiva correlacional. Se midió las percepciones de los clientes de la ONG Pro Mujer del cercado de Arequipa, respecto a las estrategias de mercadotecnia social utilizadas en el año 2014. Es correlacional, por lo que se analizó la relación entre las estrategias de mercadotecnia social y la fidelización de los clientes de la ONG Pro Mujer del cercado de Arequipa en el año 2014. Se utilizó el diseño 
transversal - no experimental, para ello se ejecutó una sola medición de las variables en estudio, y también es no experimental al no haberse realizado manipulación alguna de las variables en estudio de parte del investigador. La investigación se desarrolló en las agencias de la ONG Pro Mujer del cercado de Arequipa (Avelino, Altiplano, Zamacola y Paucarpata), con la participación activa de los clientes que mantienen relación crediticia desde el año 2013; el estudio se llevó a cabo en el mes de diciembre del año 2014. La muestra se distribuyó en 50 clientes de la agencia Avelino, 45 clientes de la agencia Altiplano, 25 clientes de la agencia Zamacola y 20 clientes de la agencia Paucarpata. El tipo de muestreo utilizado en la investigación es probabilístico, al seleccionarse los elementos muestrales aleatoriamente y estratificado con afijación proporcional, al segmentar la población por agencias, ponderando cada estrato de acuerdo al porcentaje correspondiente. Se ejecutaron encuestas personales a los clientes (mujeres) de la ONG Pro Mujer del cercado de Arequipa (Avelino, Altiplano, Zamacola, Paucarpata). Se utilizó un cuestionario estructurado, dividido en tres bloques: el primer grupo con 22 preguntas dicotómicas $(0=$ No, $1=\mathrm{Si})$, que evaluó la variable independiente "estrategias de mercadotecnia social", el segundo grupo con 22 preguntas de escala Lickert ( 1 = Muy en desacuerdo, $5=$ Muy de Acuerdo) y valoró la variable dependiente "fidelización de clientes" y un tercer grupo de 6 preguntas de información complementaria. Para el procesamiento y análisis de datos se utilizó la Hoja de Cálculo "Excel", asimismo se realizó el respectivo análisis de distribución de frecuencias con sus correspondientes gráficos estadísticos que muestran el resultado de la información lograda. Para la comprobación de la hipótesis, se realizó la prueba estadística no paramétrica del chi cuadrado, para ello se utilizó el programa estadístico SPSS V.20.

\section{RESULTADOS}

La consistencia interna se calculó con el coeficiente de alfa de Cronbach; la consistencia interna se considera aceptable cuando se encuentra entre 0,70 y 0,90 (Campo-Arias \& Oviedo, 2008).

Para la variable independiente, "Estrategias de mercadotecnia social" conformada por 22 preguntas de escala dicotómica, el valor del coeficiente fue de 0.843 .

Tabla 1. Fiabilidad

\begin{tabular}{|c|c|}
\hline $\begin{array}{c}\text { Alfa de } \\
\text { Cronbach }\end{array}$ & $\begin{array}{c}\text { N de } \\
\text { elementos }\end{array}$ \\
\hline 0,843 & 22 \\
\hline
\end{tabular}

Para la variable dependiente, "Fidelización de clientes", conformada por 22 preguntas de escala politómica, el valor del coeficiente fue de 0,902.

Tabla 2 .Fiabilidad

\begin{tabular}{|c|c|}
\hline $\begin{array}{c}\text { Alfa de } \\
\text { Cronbach }\end{array}$ & $\begin{array}{c}\text { N de } \\
\text { elementos }\end{array}$ \\
\hline 0,902 & 22 \\
\hline
\end{tabular}




\section{Contraste de las hipótesis de investigación}

a) Hipótesis de investigación $\mathbf{N}^{\circ} 01$

$\mathrm{H}_{0} \quad$ La estrategia "producto" no ha contribuido en la fidelización de clientes de las agencias de la ONG Pro Mujer del cercado de Arequipa, año 2014.

$\mathrm{H}_{1} \quad$ La estrategia "producto" si ha contribuido en la fidelización de clientes de las agencias de la ONG Pro Mujer del cercado de Arequipa, año 2014.

Tabla 3. Fidelización de clientes por Producto

\begin{tabular}{|l|c|c|c|}
\hline & No & Si & Total por Fila \\
\hline Bueno & 3 & 108 & 111 \\
\hline Regular & 4 & 25 & 29 \\
\hline Total por Columna & 7 & 133 & $\mathbf{1 4 0}$ \\
\hline
\end{tabular}

Tabla 4. Pruebas de Independencia

\begin{tabular}{|l|c|c|c|}
\hline \multicolumn{1}{|c|}{ Prueba } & Estadístico & GI & Valor-P \\
\hline $\begin{array}{l}\text { Chi-cuadrada con corrección de } \\
\text { Yates }\end{array}$ & 3.848 & 1 & $\mathbf{0 . 0 4 9 8}$ \\
\hline
\end{tabular}

Decisión: SI se rechaza la $\mathrm{H}_{0}(\mathrm{P}<0,05)$

b) Hipótesis de investigación $\mathbf{N}^{\circ} 02$

$\mathrm{H}_{0} \quad$ La estrategia "precio" no ha contribuido en la fidelización de clientes de las agencias de la ONG Pro Mujer del cercado de Arequipa, año 2014.

$\mathrm{H}_{2} \quad$ La estrategia "precio" si ha contribuido en la fidelización de clientes de las agencias de la ONG Pro Mujer del cercado de Arequipa, año 2014.

Tabla 5. Fidelización de clientes por Precio

\begin{tabular}{|l|c|c|c|}
\hline \multicolumn{1}{|c|}{ Categorías } & No & Si & Total por Fila \\
\hline Bueno & 9 & 102 & 111 \\
\hline Regular & 5 & 24 & 29 \\
\hline Total por Columna & 14 & 126 & $\mathbf{1 4 0}$ \\
\hline
\end{tabular}

Tabla 6. Pruebas de Independencia

\begin{tabular}{|l|c|c|c|}
\hline \multicolumn{1}{|c|}{ Prueba } & Estadístico & GI & Valor-P \\
\hline $\begin{array}{l}\text { Chi-cuadrada con corrección de } \\
\text { Yates }\end{array}$ & 1.237 & 1 & $\mathbf{0 . 2 6 6 0}$ \\
\hline
\end{tabular}

Decisión: NO se rechaza la $\mathrm{H}_{0}(\mathrm{P}>0,05)$ 
c) Hipótesis de investigación $\mathbf{N}^{\circ} 03$

$\mathrm{H}_{0} \quad$ La estrategia "promoción" no ha contribuido en la fidelización de clientes de las agencias de la ONG Pro Mujer del cercado de Arequipa, año 2014.

$\mathrm{H}_{3} \quad$ La estrategia "promoción" si ha contribuido en la fidelización de clientes de las agencias de la ONG Pro Mujer del cercado de Arequipa, año 2014.

Tabla 7. Fidelización de clientes por Promoción

\begin{tabular}{|l|c|c|c|}
\hline & No & Si & Total por Fila \\
\hline Bueno & 20 & 91 & 111 \\
\hline Regular & 4 & 25 & 29 \\
\hline Total por Columna & 24 & 116 & $\mathbf{1 4 0}$ \\
\hline
\end{tabular}

Tabla 8. Pruebas de Independencia

\begin{tabular}{|l|c|c|c|}
\hline \multicolumn{1}{|c|}{ Prueba } & Estadístico & GI & Valor-P \\
\hline $\begin{array}{l}\text { Chi-cuadrada con corrección de } \\
\text { Yates }\end{array}$ & 0.068 & 1 & $\mathbf{0 . 7 9 4 2}$ \\
\hline
\end{tabular}

Decisión: NO se rechaza la $\mathrm{H}_{0}(P>0,05)$

\section{d) Hipótesis de investigación $\mathbf{N}^{\circ} 04$}

$\mathrm{H}_{0} \quad$ La estrategia "distribución" no ha contribuido en la fidelización de clientes de las agencias de la ONG Pro Mujer del cercado de Arequipa, año 2014.

$\mathrm{H}_{4} \quad$ La estrategia "distribución" si ha contribuido en la fidelización de clientes de las agencias de la ONG Pro Mujer del cercado de Arequipa, año 2014.

Tabla 9. Fidelización de clientes por Distribución

\begin{tabular}{|l|c|c|c|}
\hline & No & Si & Total por Fila \\
\hline Bueno & 7 & 104 & 111 \\
\hline Regular & 3 & 26 & 29 \\
\hline Total por Columna & 10 & 130 & $\mathbf{1 4 0}$ \\
\hline
\end{tabular}

Tabla 10. Pruebas de Independencia

\begin{tabular}{|l|c|c|c|}
\hline \multicolumn{1}{|c|}{ Prueba } & Estadístico & GI & Valor-P \\
\hline $\begin{array}{l}\text { Chi-cuadrada con corrección de } \\
\text { Yates }\end{array}$ & 0.120 & 1 & $\mathbf{0 . 7 2 8 6}$ \\
\hline
\end{tabular}

Decisión: NO se rechaza la $\mathrm{H}_{0}(\mathrm{P}>0,05)$ 


\section{Contraste de la hipótesis general}

Tabla 11. Consolidado de las pruebas de hipótesis específicas

\begin{tabular}{|c|c|c|}
\hline $\begin{array}{c}\text { Hipótesis } \\
\text { específica }\end{array}$ & Valor- $P$ & \multicolumn{1}{c|}{ Decisión } \\
\hline $\mathrm{N}^{\circ} 01$ & $\mathrm{P}=0,0498$ & SI se rechaza la $\mathrm{H}_{0}(\mathrm{P}<0,05)$ \\
\hline $\mathrm{N}^{\circ} 02$ & $\mathrm{P}=0,2660$ & $\mathrm{NO}$ se rechaza la $\mathrm{H}_{0}(\mathrm{P}>0,05)$ \\
\hline $\mathrm{N}^{\circ} 03$ & $\mathrm{P}=0,7942$ & $\mathrm{NO}$ se rechaza la $\mathrm{H}_{0}(\mathrm{P}>0,05)$ \\
\hline $\mathrm{N}^{\circ} 04$ & $\mathrm{P}=0,7286$ & $\mathrm{NO}$ se rechaza la $\mathrm{H}_{0}(\mathrm{P}>0,05)$ \\
\hline
\end{tabular}

Fuente: Cuadro $\mathrm{N}^{\circ} 15$, Cuadro $\mathrm{N}^{\circ} 17$, Cuadro $\mathrm{N}^{\circ} 19$, Cuadro $\mathrm{N}^{\circ} 21$

Elaboración propia.

\section{Decisión:}

De las cuatro hipótesis específicas, sólo la hipótesis específica $N^{0} 01$ rechaza la hipótesis nula, y las hipótesis especificas $\mathrm{N}^{\circ} 02, \mathrm{~N}^{\circ} 03$ y $\mathrm{N}^{\circ} 04$, no rechazan su hipótesis nula respectiva, por lo que se Acepta la Hipótesis General.

\section{CONCLUSIONES}

a) La estrategia "producto" de las agencias de la ONG Pro Mujer del cercado de Arequipa, en el año 2014, si ha contribuido en la fidelización de sus clientes, debido a que este indicador, obtiene un valor-P de 0,0498 $(P<0.05)$, lo que demuestra que si existe relación entre las variables.

b) La estrategia "precio" de las agencias de la ONG Pro Mujer del cercado de Arequipa, en el año 2014, no ha contribuido en la fidelización de sus clientes, debido a que este indicador, obtiene un valor-P de 0,2660 ( $P>0.05)$, lo que demuestra que no existe relación entre las variables.

c) La estrategia "promoción" de las agencias de la ONG Pro Mujer del cercado de Arequipa, en el año 2014, no ha contribuido en la fidelización de sus clientes, debido a que este indicador, obtiene un valor-P de 0,7942 ( $P>0.05)$, lo que demuestra que no existe relación entre las variables.

d) La estrategia "distribución" de las agencias de la ONG Pro Mujer del cercado de Arequipa, en el año 2014, no ha contribuido en la fidelización de sus clientes, debido a que este indicador, obtiene un valor-P de 0,7286 ( $P>0.05)$, lo que demuestra que no existe relación entre las variables.

e) Las estrategias de mercadotecnia social de las agencias de la ONG Pro Mujer del cercado de Arequipa, en el año 2014, no han contribuido en la fidelización de sus clientes, por lo tanto se comprueba la Hipótesis General. 


\section{REFERENCIAS BIBLIOGRÁFICAS}

- Andrea, A. (1995). Marketing Social Change. San Francisco: Jossey-Bass Publishers.

- Andreasen, A. (1995). Marketing Social Change: Changing Behavior Promote Health, Social Development, and the Enviroment.San Francisco: Jossey-Bass Publishers.

- Arellano, R. (2010). Marketing: Enfoque América Latina . Lima: Pearson Educación.

- Barquero, J. (2007).Marketing de clientes: Quien se ha llevado a mi cliente. Madrid: Mc Graw Hill.

- Bastos, A. (2006). Fidelización del cliente. Introducción a la venta personal y a la direacción de ventas. España: Vigo.

- Belch, G., \&Belch, M. (2004). Publicidad y Promoción: Perspectiva de la Comunicación de Marketing Integral. México: Mc Graw Hill.

- Bernal, L., \& Hernandez, D. (2008). Marketing social en organizaciones lucrativas: ¿Imagen pública o apoyo a la comunidad? Bogotá: Pontifica Universidad Javeriana.

- Bizzinco. (2014). Bizzinco. Recuperado el 25 de Setiembre de 2014, de Capacitación empresarial: http://www.bizzinco.com/capacitacion-empresarial-ver-definicion-3141

- Dorrian, M., \& Lucas, G. (2006). Publicidad de Guerrilla: Otras formas de comunicar. Madrid: Gustavo Gili.

- E-CONOMIC. (2010). e-conomic. Recuperado el 26 de Setiembre de 2014, de Definición de crédito: http://www.e-conomic.es/programa/glosario/definicion-credito

- FIDELIZARCLIENTES.ES. (2014). Fidelizarclientes.es. Recuperado el 27 de Setiembre de 2014, de Curso de fidelización: http://www.fidelizarclientes.es/curso/cap01.htm

- Fischer, L., \& Espejo, J. (2004). Mercadotecnia. México: Mc Graw Hill.

- Forero, J. (2009). El marketing social como estrategia para la promoción de salud. La Sociología en sus escenarios, 22.

- Gallardo, L. (2012). Hacia un modelo comunicativo para obtener prácticas emancipadoras en la sociedad postindustrial. Questión. Revista Especializada en Periodismo y Comunicación, 145-147.

- Grier, S., \& Bryant, C. (2004). Anuario de Salud Pública. San Francisco: Universidad de California.

- Kotler, P. (2001). Dirección de Mercadotecnia. México Distrito Federal: Pearson Educación.

- Kotler, P., \& Roberto, E. (1992). Marketing social: estrategias para cambiar la conducta pública. Madrid: Ediciones Díaz de Santos.

- Kotler, P., \& Zaltamn, G. (1971). Marketing Social: Una aproximación al cambio social planificado. Journal de Marketing, 3-12.

- Kotler, P., Roberto, N., \& Nancy, L. (2002). Marketing Social: Mejorando la calidad de vida. California: SAGE.

- Mendive, D. (2008). Marketing social. Manual Práctico. Buenos Aires: De los cuatro vientos.

- Mendive, D. (19 de Marzo de 2011). Marketing Social. Recuperado el 25 de Julio de 2015, de El marketing social en las ONG's: https://marketingsocial.wordpress.com/2011/03/19/el-marketing-social-en-lasong\%C2\%B4s/

- Mujeres en Red. (Enero de 2007). Mujeres en Red. Recuperado el 25 de Setiembre de 2014, de Qué significa empoderamiento: http://www.mujeresenred.net/spip.php?article1307

- ONG PRO Mujer. (2010). ONG Pro Mujer. Recuperado el 24 de Setiembre de 2014, de Cómo lo hacemos: http://promujer.org/espanol/que-hacemos/como-lo-hacemos/ 
- Pinilla, M. (2011). Contribuciones del marketing social a la sostenibilidad de fundaciones sin ánimo de lucro: un estudio de caso. Manizales: Universidad Nacional de Colombia.

- Salazar, J. (2008). Desarrollo de una estrategia de marketing social orientada al incremento de competencias empresariales de las MYPE de la Región Cerro de Pasco. Lima: Universidad Nacional Mayor de San Marcos.

- Smithe, W. (11 de Setiembre de 2007). SAGE publicaciones. Recuperado el 24 de Setiembre de 2014, de http://www.sagepub.com/upmdata/19260_Chapter_1_Defining_Social_Marketing.pdf

- Stanton, W., Etzel, M., \& BRUCE, W. (2004). Fundamentos de marketing. México: Mc Graw Hill.

- Vivir en salud. (2013). Vivir en salud. Recuperado el 25 de Setiembre de 2014, de Noticias: http://www.vivirensalud.com/noti_vivir.asp

- Weinrich, N. (1999). Hands-On Social Marketing.California: SAGE publications.

- WIKIPEDIA. (17 de Setiembre de 2014). Wikipedia Responsabilidad Social Corporativa. Recuperado el 28 de Setiembre de 2014, de http://es.wikipedia.org/wiki/Responsabilidad_social_corporativa

- Zambrana, M. (2012). Universidad Complutense de Madrid. Recuperado el 25 de Julio de 2015, de Editorial IEPALA y Librería Tercer Mundo: http://www.iepala.es/IMG/pdf/ADJUNTO_Tesina_Marketing_Social_Libreria_Tercer_Mu ndo.pdf

Recibido : 21/4/2016

Aceptado para publicación

5/5/2016 